\title{
Performance enhancement of overlapping BSSs via dynamic transmit power control
}

\author{
Xiaoying Lei* and Seung Hyong Rhee
}

\begin{abstract}
In densely deployed wireless local area networks (WLANs), overlapping basic service sets (BSSs) may suffer from severe performance degradations. Mobile stations in a BSS may compete for channel access with stations that belong to another BSS in such environment, and it reduces overall throughput due to the increased collision probability. In this paper, we propose a new scheme for transmit power control, which enables mobile stations to dynamically adjust their transmit powers. Using our mechanism, those stations in different BSSs will have more chances of simultaneous transmissions and thus improve their performances by enhancing spatial reuse. We develop a Markov chain model to analyze the performance of the proposed scheme and also perform extensive simulations. Both the analytical and simulation results show that our mechanism effectively improves the network performance of WLANs.
\end{abstract}

Keywords: IEEE 802.11 MAC; Overlapping BSSs; Interference; Transmit power

\section{Introduction}

As IEEE 802.11 wireless local area networks (WLANs) have been widely deployed in homes, offices, and public places [1], the high density of WLANs has posed a great concern on the problem of co-channel interferences. Thus, the overall network performance of WLANs may be severely degraded unless an efficient scheme is provided to reduce the interference.

A WLAN basic service set (BSS) is typically formed by an access point (AP) and a number of stations associated with the AP [2], and in that case, data transmissions are allowed only between the stations and the AP. When the coverage of nearby co-channel BSSs overlaps with each other, they are called overlapping BSSs (OBSSs) [3]. In case a station located at the overlapping area transmits frames, other stations of the neighbor BSS may sense the transmission and refrain their transmissions. Also if they cannot sense the transmission, then they will become hidden terminals to the transmitter. Therefore, the chance of simultaneous transmissions among OBSSs is reduced, and thus the whole network may suffer from the poor spatial reuse of OBSSs.

*Correspondence: wodeying21@kw.ac.kr

Department of Electronics Convergence Engineering, Kwangwoon University, 447-1, Wolgye Dong, Nowon-GU, Seoul, Korea
Many solutions have been suggested so far to dynamically control the transmit power of WLAN stations and thus to improve the overall throughput of the network [1,4-8]. By adopting those schemes, stations are able to reduce their transmission ranges using only proper amount of transmit power, such that more stations can simultaneously transmit and thus the overall throughput is increased. The previous works, however, may not be adopted in a practical WLAN system: For example, the problem of how to determine the proper power level is not fully investigated in [1,4]. Also [6] is based on assumptions that may not be possible in real world, [7] requires the real-time adaptation of a measurement algorithm, and power control scheme in [8] is limited to use only in ad hoc mode.

In this paper, we propose a method for dynamic transmit power control to enhance the throughput of OBSSs. First, we study the four different radio ranges in 802.11 systems and how OBSSs interfere with each other in a density WLAN. Based on these observations, we propose a new power control scheme such that every station keeps a table for recording the path loss between itself and the neighbor BSS stations from which request to send/clear to send (RTS/CTS) frames can be overheard. Utilizing the information, those stations adjust their transmit powers and data frames are delivered using only the proper powers. We develop a discrete-time Markov chain model in

\section{照 Springer}

(C) 2015 Lei and Rhee; licensee Springer. This is an Open Access article distributed under the terms of the Creative Commons Attribution License (http://creativecommons.org/licenses/by/4.0), which permits unrestricted use, distribution, and reproduction in any medium, provided the original work is properly credited. 
order to verify that our proposed method provides the OBSSs with more opportunities of simultaneous transmissions and thus increases spatial reuse. In addition, simulation results are presented to validate our proposed scheme and its analytical model.

The remaining part of this paper is organized as follows. We discuss the related previous works and study the interference occurred in OBSSs in Section 2. The details of our proposed power control method are addressed in Section 3, and the Markov chain model is investigated in Section 4. The extensive simulation results are reported in Section 5, and finally, concluding remarks are drawn in Section 6.

\section{Problem definition and related works}

\subsection{Problem definition}

There are four different radio ranges in 802.11 systems as illustrated in Figure 1 [9]: transmission range, net allocation vector (NAV) set range, clear channel assessment (CCA) busy range, and interference range. Transmission range is the range from a transmitter $(\mathrm{T})$ and represents the area within which the receiver station (R) can receive a frame successfully. The NAV set range is the area within which the wireless stations (A, B) can set the NAVs correctly, based on the duration/ID information carried in the RTS/CTS frames. CCA busy range is the area within which the wireless stations $(C, D)$ can physically sense the
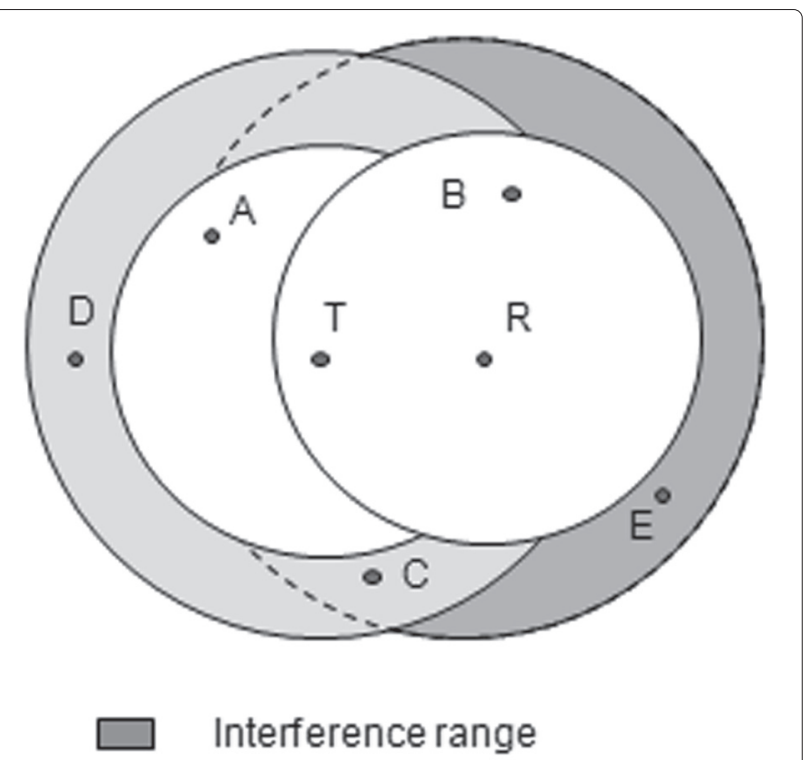

\section{CCA busy range}

\section{NAV set range}

Figure $1 \mathrm{~A}$ sketch of the radio ranges during a four-way frame exchange [9]. busy channel during the data transmission. Interference range is the range from a receiver and represents the area within which the wireless stations (E) are able to interfere with the reception of data frames at the receiver.

Currently most stations are configured to transmit at their maximum powers, and such a default deployment may result in a high interference among OBSSs [1]. A scenario of interference among OBSSs is illustrated in Figure 2, where two BSSs, $\mathrm{BSS}_{1}$ and $\mathrm{BSS}_{2}$, overlap with each other. When station $\mathrm{A}$, which belongs to $\mathrm{BSS}_{1}$ and locates at the overlapping area, begins a transmission, neighbor $\mathrm{AP}$ (i.e., $\mathrm{AP}_{2}$ ) and other stations (such as $\mathrm{B}$ ) will sense the transmission and set their NAVs. Also other neighbor stations which are hidden terminals to the sender A, e.g., D and E, may try to access channel. In case $\mathrm{D}$ successfully transmits a data frame to $\mathrm{AP}_{2}$, the AP cannot response with an ACK in time since it has set NAV. Due to the unsuccessful transmission, D increases its contention window and contends for retransmission. In this example, we can see that transmission from one BSS can hamper the operation of neighbor BSSs. This problem of the 802.11 WLANs comes from the fact that each station must rely on its direct experience in estimating congestion, which often leads to asymmetric views [10].

\subsection{Related works}

Several attempts have been made to improve the performance of 802.11 MAC by utilizing transmit power control scheme. Since the transmit power control (TPC) method standardized in the IEEE 802.11 suffers from inaccuracies, Oteri et al. [4] propose a fractional CSMA/CA scheme by combining the TPC with user grouping and inter-BSS coordination to improve the performance of overlapping BSSs. However, their approach lacks of a mechanism for determining the proper transmit power. In [6], an iterative power control algorithm is proposed to increase the number of concurrent transmissions in the dense wireless networks. This proposal is based on the assumptions that every node has complete knowledge of the network topology and current configuration, which may not be possible in real world.

In [7], a run-time self-adaptation algorithm is proposed based on packet loss differentiation, which can jointly adapt both transmit power and physical carrier sensing (PCS) threshold. The problem of this scheme is that it requires metrics such as PER and interference level to be measured in real time which can increase the burden of system. Also Cesana et al. [8] present an interference aware MAC for ad hoc mode network, in which each station transmits using RTS/CTS procedure, and the information about reception powers of RTS frames and interference levels is inserted into CTS packets. Utilizing the information, stations which overhear a CTS can 


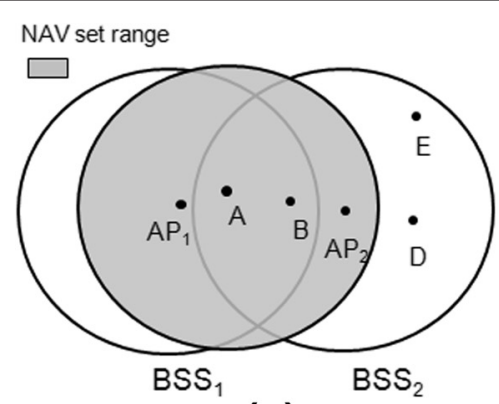

(a)

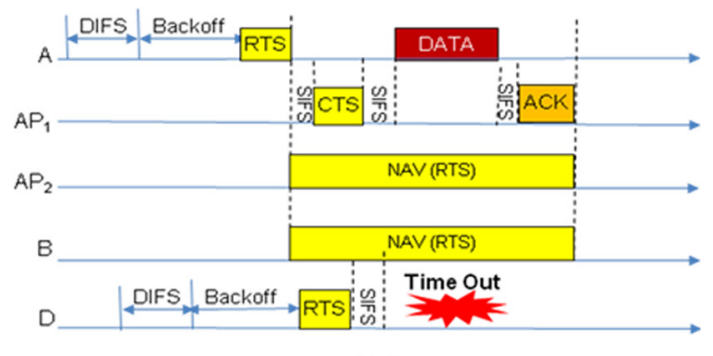

(b)

Figure 2 A simple scenario of OBSSs. (a) Topology of OBSSs and (b) transmission process for OBSSs.

tune their transmit powers such that they can transmit simultaneously without interfering with each other.

For performance enhancement of OBSSs, many recent works have provided different approaches. Li et al. [11] propose an interference avoidance algorithm to mitigate the interference from the neighbor BSS operating at the same channel. However, this scheme enables AP to drop its defer threshold to energy detect threshold when transmitting to stations located at overlapping area. Thus a hidden terminal to AP can sense the transmission from $\mathrm{AP}$ and the collision probability is reduced. Fang et al. [12] propose a PCF-based two-level carrier sensing mechanism which adopts two NAVs in stations, namely self BSS network NAV (SBNAV) and OBSSs network NAV (OBNAV). When a transmission processes in one of the BSSs, the station which senses it sets the value of its NAV to be either SBNAV or OBNAV, whichever is bigger. If there are no OBSSs, the OBNAV is set to 0. In [13], an interference packet detection scheme in link layer is proposed, in which a receiving station that detects interference packets reports the existence of another BSS to AP. Then the AP announces channel switching to all stations in its BSS for avoidance of interference. There is no guarantee that the chosen channel is free from interference though.

\section{Dynamic transmit power control}

Our proposed dynamic TPC (DTPC) scheme is presented in this section. In the DTPC, the stations located at overlapping area are referred as interference prone (IP) stations adopting the notion in [11]. As all the stations continually monitor the ongoing transmissions, combining with the information recorded in the path loss table, a station can determine whether it can start a concurrent transmission. Then all the stations which try to start concurrent transmissions adjust their transmit powers to proper levels and compete for channel access. If one station is successful to access the channel, then since its transmission uses a low power, more stations may become hidden terminals to the transmitter. Thus we propose all the stations to use RTS/CTS procedure where the
RTS/CTS frames are exchanged using their maximum powers.

Our DTPC scheme enables the performance enhancement in two aspects: First, when a transmission from an IP station is ongoing, another station which belongs to a neighbor BSS and is not a hidden terminal to the IP station can start a simultaneous transmission after tuning the transmit power. Second, if a hidden terminal starts a transmission in parallel with the IP station, the neighbor AP can adjust its transmit power for timely ACK response, which means a successful transmission.

\subsection{NAV reset timer modification}

A timer named RESET_NAV is defined in the IEEE 802.11 MAC for NAV update [14]. The stations overhearing an RTS set their NAVs and also set the timer RESET_NAV with a duration of CTS_Time+2SIFS_Time + 2Slot_Time. Here, CTS_Time is calculated from the length of the CTS frame and the rate at which the CTS frame is transmitted. After setting the timer, the stations will reset their NAVs if they overhear DATA frame from the RTS sender.

As the RTS/CTS frames are transmitted on the maximum power and data frames are transmitted on a tuned power, some stations which set NAVs according to an RTS frame may not overhear the data frame, and the timer of these stations will expire within the time-out. We modify the NAV reset timer as follows: A new timer D_RESET_NAV is added, and the duration of this timer is the same as the duration field of RTS. Thus if a station overhears an RTS of a station that belongs to its BSS, it sets D_RESET_NAV, otherwise it sets RESET_NAV. It makes sense because in 802.11 WLAN, a station is supposed to receive all the incoming frames and at least decode the MAC header part unless it is in the sleeping mode. Moreover, in infrastructure architecture, the direct transmission is only possible between AP and stations. Thus, a station can check the address fields of a received packet to confirm whether the sender belongs to the domestic BSS. This modification in the NAV reset timer guarantees the domestic stations which set D_RESET_NAV timer will 
not experience time-out until the ongoing transmission terminates. Our DTPC proposes that after RESET_NAV timer expires, stations enter into the back-off (BO) process directly. The station whose $\mathrm{BO}$ counter decreases to 0 will access the channel.

\subsection{Path loss recording}

In our proposal, an AP broadcasts the value of an allowable maximum transmit power via beacon frames, and other stations transmit RTS/CTS frames using the maximum power. Also it is assumed that all BSSs adopt a same value of maximum transmit power. Two more fields are added into the RTS frame, reception power and signal to interference and noise (SINR). When a station transmits an RTS frame on maximum power, it piggybacks the reception power of the beacon it received recently and SINR of the beacon. Note that

$$
S I N R_{j}=\frac{P_{t r a} \times G_{i j}}{N_{j}},
$$

where $P_{\text {tra }}$ is the transmit power of sender $i, G_{i j}$ is the path loss between sender $i$ and receiver $j$, and $N_{j}$ is the noise and interference experienced in $j$. Thus when the AP receives the RTS, it can calculate the path loss from the sender. Also a neighbor BSS station which overhears the RTS packets can calculate the path loss between the sender and itself, by adopting the allowable maximum transmit power. As the RTS/CTS frames are exchanged on the maximum power, it prevents hidden terminals and exposed terminals in a wide range. After the RTS/CTS frame exchanged, the sender adjusts its transmit power to a low level and delivers a data frame SIFS later.

In DTPC, each station keeps a table for recording reception power of beacon frame and path loss between itself and the neighbor BSS stations from which it can overhear an RTS/CTS frame, i.e. $\left\langle\right.$ node $_{i d}$, pathloss $i j, p_{\text {rev }}>$. AP keeps a table for its own BSS stations and neighbor BSS stations located at overlapping area. When a station overhears an RTS/CTS frame, it updates the record related to the sender. If there is no record for the sender, it adds a new record into the table.

\subsection{Tuning transmit power}

In this section, the method for tuning transmit power is explained. We assume the thresholds $\operatorname{SINR}(\gamma)$ for stations in all BSSs are the same. A BSS that first starts a transmission is called primary BSS, and the other BSS overlapping with the primary BSS is referred as a secondary BSS. Let $P_{i \_r e}$ be the power that station $i$ which belongs to primary BSS received a beacon from its AP, and $P_{j_{-} t r}$ be the transmit power of station $j$ in secondary BSS. Also let $I_{i}$ be the noise and interference that station $i$ experienced, $G_{i j}$ be the path loss between station $i$ and station $j$ assuming a symmetric channel, and let $\operatorname{SINR}_{i}$ be the SINR that $i$ experienced when it received a frame from its AP. In order to guarantee that transmissions from $j$ does not disturb ongoing transmission in $i$, the following condition is required.

$$
\begin{gathered}
\frac{P_{i \_r e}}{I_{i}+\frac{P_{j_{-} t r}}{G_{i j}}}>\gamma . \\
\text { As } I_{i}=\frac{P_{i \_r e}}{\operatorname{SINR}_{i}}, \text { after rearrangement of }(1) \text {, we can get } \\
P_{j_{-} t r}<\frac{P_{i_{-} r e} \cdot G_{i j}\left(\operatorname{SINR}_{i}-\gamma\right)}{\operatorname{SINR}_{i} \cdot \gamma} .
\end{gathered}
$$

In order to guarantee the transmission from station $j$ can be received by its AP successfully, the condition below should be satisfied:

$$
\frac{P_{j_{-} t r} \times G_{A P \_j}}{I_{A P}}>\gamma .
$$

Rearranging (3) gives

$$
P_{j_{-} t r}>\frac{I_{A P} \times \gamma}{G_{A P \_j}},
$$

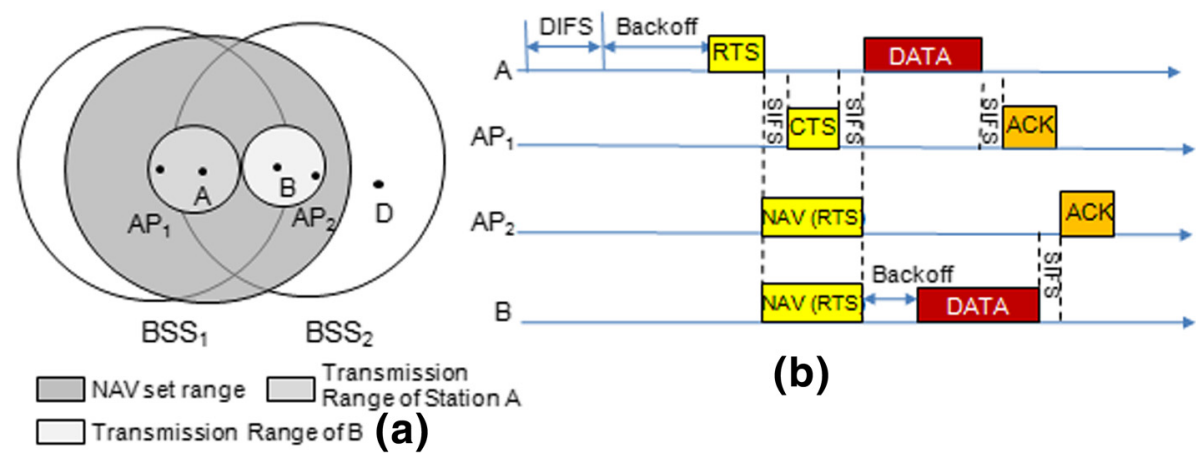

Figure 3 Proposed scheme works in a non-hidden terminal environment. (a) Radio range and (b) transmission process. 


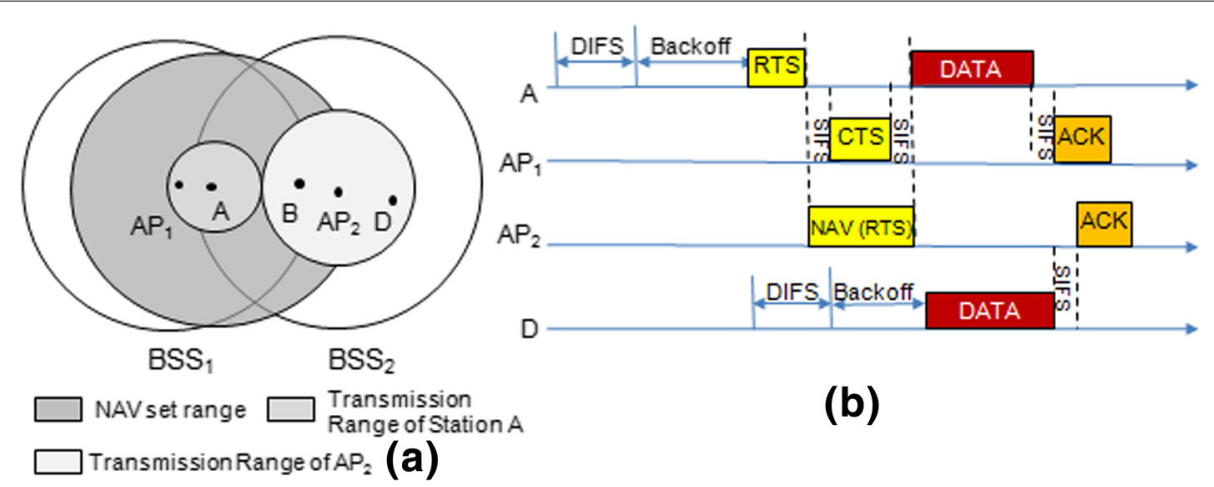

Figure 4 Proposed scheme works in a hidden terminal environment. (a) Radio range and (b) transmission process.

where $\mathrm{G}_{A P_{-} j}$ is the path loss between station $j$ and its AP. Combining (2) and (4), the transmit power of station $j$ can be adjusted as follows:

$$
\frac{I_{A P} \times \gamma}{G_{A P \_j}}<P_{j_{-} t r}<\frac{P_{i_{-} r e} \cdot G_{i j}\left(\operatorname{SINR}_{i}-\gamma\right)}{\operatorname{SINR}_{i} \cdot \gamma} .
$$

\subsection{Transmissions in a non-hidden terminal environment} We consider the proposed DTPC in a non-hidden terminal environment. The network topology is given in Figure 2a, and we use Figure $3 \mathrm{a}$ to illustrate the radio ranges of stations while using Figure $3 \mathrm{~b}$ to present the transmission process. As shown in Figure 3b, the RTS frame of station A contains the reception power of the recently received beacon from $\mathrm{AP}_{1}$ and SINR of the beacon. The stations which belong to $\mathrm{BSS}_{2}$ and overhear this transmission (e.g., station $\mathrm{B}$ and $\mathrm{AP}_{2}$ ) will set their NAVs and RESET_NAV timers. After A receives a CTS from $\mathrm{AP}_{1}$, it tunes its transmit power and transmits a data frame. The stations which set NAVs according to the RTS frame but cannot sense the following data frame will experience the RESET_NAV timer's time-out and enter into the $\mathrm{BO}$ process. Station B, whose BO counter reaches 0 first, accesses the channel and delivers a data frame after adjusting its transmit power.



Figure 5 State transition diagram.

\subsection{Transmissions in a hidden terminal environment}

Now we use Figure $4 \mathrm{a}$ and Figure $4 \mathrm{~b}$ to consider the transmission process of DTPC in a hidden terminal scenario. The network topology is the same as depicted in Figure 2a. When station A transmits an RTS frame, $\mathrm{AP}_{2}$ overhears it and sets its NAV as shown in Figure 4b. Then after receiving a CTS frame from $\mathrm{AP}_{1}$, station $\mathrm{A}$ adjusts its power level and transmits its data frame on a possible low power. As $\mathrm{AP}_{2}$ cannot overhear this data frame, its RESET_NAV timer expires and it will not set NAV when station A is exchanging data frame with $\mathrm{AP}_{1}$. $\mathrm{D}$ which is a hidden terminal to station $\mathrm{A}$, after sensing the channel is idle, transmits a data frame to $\mathrm{AP}_{2}$ during A's ongoing transmission. $\mathrm{AP}_{2}$ adjusts its power level based on (5) and then responses with an ACK SIFS later.

\section{Performance analysis}

In order to analyze the performance of the proposed scheme compared to 802.11 MAC, we develop an analytical model using the discrete-time Markov chain in this section.

\subsection{Markov chain model}

While an ongoing transmission in a BSS prevents transmissions in a neighbor OBSS in the legacy 802.11 MAC, in our proposed scheme, however, the OBSSs are allowed to transmit simultaneously. Thus, in order to compare the channel utilization of the proposed scheme (DTPC) and the legacy MAC, we make an assumption that the cochannel is divided into two sub channels, and each BSS

\section{Table 1 System parameters}

\begin{tabular}{llc}
\hline Parameters & Value \\
\hline$N$ & Number of sub channels & 2 \\
$p$ & Transmission probability & 0.05 \\
$q$ & Termination probability & 0.1 \\
$C$ & Channel bit rate & $54 \mathrm{Mbps}$ \\
\hline
\end{tabular}




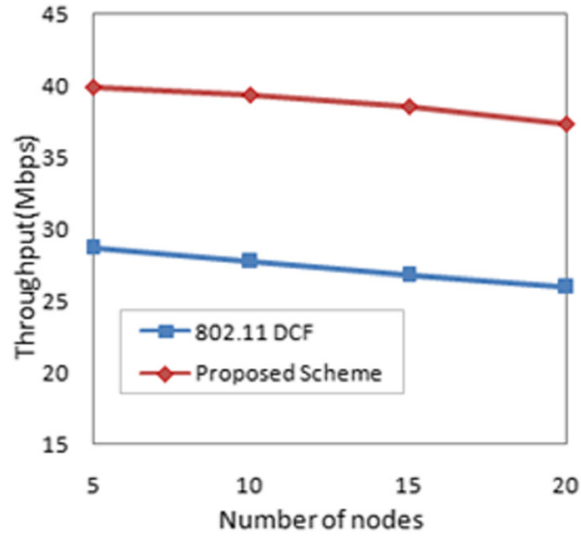

(a)

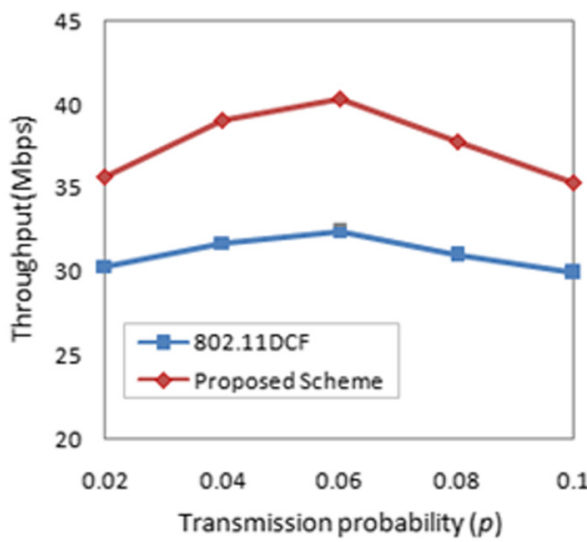

(b)

Figure 6 Analysis results. (a) Throughput vs. number of stations; (b) throughput vs. transmission probability.

may occupy one of them. Adopting slotted time, in order to make the model Markovian, we suppose that the packet lengths which are integer multiples of slot durations are independent and geometrically distributed with parameter $q$ (i.e., packet duration has a mean of $1 / q$ slots) [15]. Also we assume that devices always have packets to send to AP in each time slot, and each device attempts to transmit with probability $p$. In addition, it is assumed that there are no hidden or exposed terminals in domestic BSS.

Let $X_{n}$ be the number of transmissions ongoing in the two sub channels at time $n$. Since each BSS can process one transmission during a time slot, the state space for the model is given by $S=\{0,1,2\}$. Note that the value of $X_{n}$ can be 2 only when two BSSs process a transmission at the same slot. The relationship between $X_{n+1}$ and $X_{n}$ can be written as follows:

$$
X_{n+1}=X_{n}+S_{n}-T_{n}, n \geq 0,
$$

where $S_{n}$ is the number of new transmissions successfully started at time $n$, and $T_{n}$ is the number of terminations at time $n$. Note that $S_{n}=1$ if a new transmission starts successfully in time slot $n$ and $S_{n}=0$, otherwise. If $X_{n}=2$, which means that both BSSs are processing transmissions, then $S_{n}=0$ with probability 1 . The number of terminations $T_{n}$ at time $n$ ranges from 0 to $X_{n}$. If $X_{n}=0$, then $T_{n}=0$ with probability 1 .

When a station has a frame to transmit, it attempts to transmit with probability $p$. If $k$ stations are transmitting in the current slot, then the success probability $D_{k}$ in the next time slot is

$$
D_{k}=K p(1-p)^{L-1},
$$

where $L$ is the number of stations. Also the probability $R_{k}^{(j)}$ that $j$ transmissions are finished when the system is in state $k$ is given by

$$
\begin{aligned}
R_{k}^{(j)} & =\operatorname{Pr}\left[j \text { transfers terminate at time } t \mid X_{t-1}=k\right] \\
& =\left(\begin{array}{l}
k \\
j
\end{array}\right) q^{j}(1-q)^{k-j} .
\end{aligned}
$$

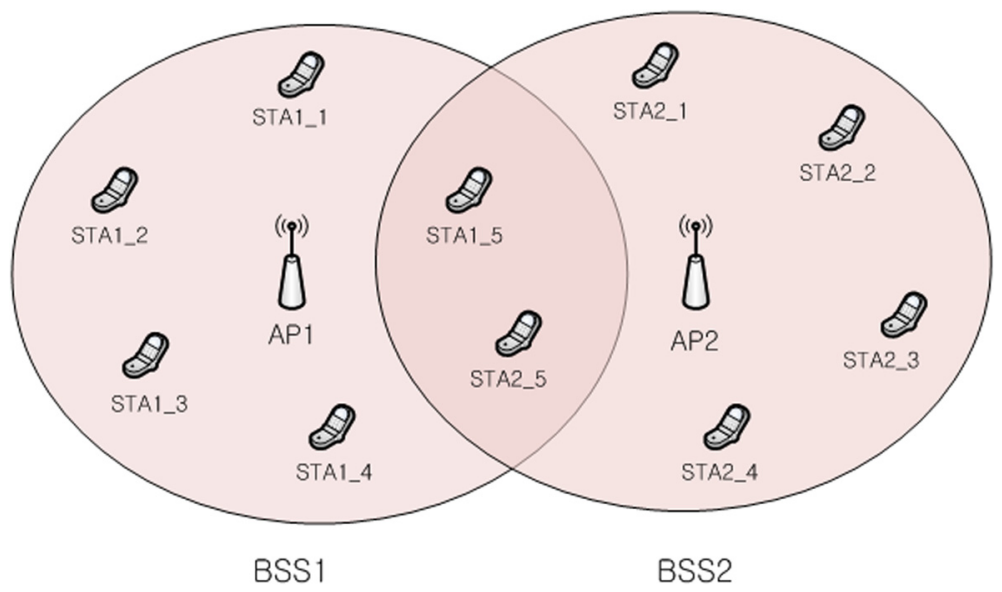

Figure 7 Network topology. 
Table 2 Simulation parameters

\begin{tabular}{lc}
\hline Parameters & Value \\
\hline Transmission rate & $54 \mathrm{Mbps}$ \\
Path loss exponent & 2 \\
SINR threshold & $2.5 \mathrm{~dB}$ \\
Maximum transmit power & $0.1 \mathrm{~W}$ \\
Normal transmit power & $0.005 \mathrm{~W}$ \\
\hline
\end{tabular}

Now the transmission probability matrix for the model can be computed as follows:

$\mathbf{P}=\left[\begin{array}{ccc}1-D_{0}\left(1-D_{0}\right)-D_{0} D_{0} & D_{0}\left(1-D_{0}\right) & D_{0} D_{0} \\ 1-D_{0} D_{1} R_{1}^{(1)}-D_{1} R_{1}^{(0)} & D_{1} R_{1}^{(0)}\left(1-D_{0}\right)+D_{0} D_{1} R_{1}^{(0)} & D_{0} D_{1} R_{1}^{(0)} \\ R_{2}^{(2)} & R_{2}^{(1)} & R_{2}^{(0)}\end{array}\right]$.

The state transition diagram of the Markov chain model for OBSS operations is depicted in Figure 5. In the developed Markov model, the legacy 802.11 MAC and our proposed DTPC differ only in transition probability. Detailed transition probabilities of both cases are omitted here due to the space limitation.

\subsection{Capacity analysis}

The average utilization $\rho$ per sub channel can be obtained as

$$
\rho=\frac{\sum_{i \in s} i \pi_{i}}{N},
$$

where $\pi_{i}$ is the limiting probability that the system is in state $i$ and $S$ is the state space of the Markov chain. Then the overall system throughput is given by

$$
T H=N \times C \times \rho,
$$

where $C$ is the channel bit rate. We study the performance of our proposed scheme compared to that of the legacy MAC using the parameters shown in Table 1.
Figure 6a shows the variation of whole network throughput according to the number of stations in each BSS. Throughputs of both proposed DTPC and the legacy 802.11 MAC decrease as the number of stations increases. The figure proves the effectiveness of our proposed scheme in enhancing the throughput. One can see that the throughput can be increased by around 40 Mbps. Figure $6 \mathrm{~b}$ shows the dependency of the throughput on the transmission probability. The throughputs of both schemes reach their peaks when the transmission probability is around 0.06 . The figure proves once again that our proposed scheme improves the network throughput.

\subsection{Additional issues}

In the Markov chain model, we have assumed that there are no hidden or exposed stations in the domestic BSS and a transmission is completed successfully. In a practical network, however, the hidden and/or exposed stations may exist and they will introduce collisions. In order to make the analysis more accurate, our future works will include the study on how to model the probability that a transmission completed successfully in a time slot.

Also, we have analyzed two overlapping BSSs. Modeling the performance of multiple OBSSs, however, becomes more challenging, as the transition probabilities in both legacy scheme and proposed solution are dependent on the network topologies. Especially, based on the location of a transmitting station, the possibility whether a neighbor BSS can process a concurrent transmission in a time slot becomes more complex. We plan to investigate this issue in our future work.

\section{Simulations}

We conduct extensive simulations of proposed DTPC using OPNET. The network size is set to $300 \times 300 \mathrm{~m}^{2}$ and two overlapping BSSs are processing their transmissions

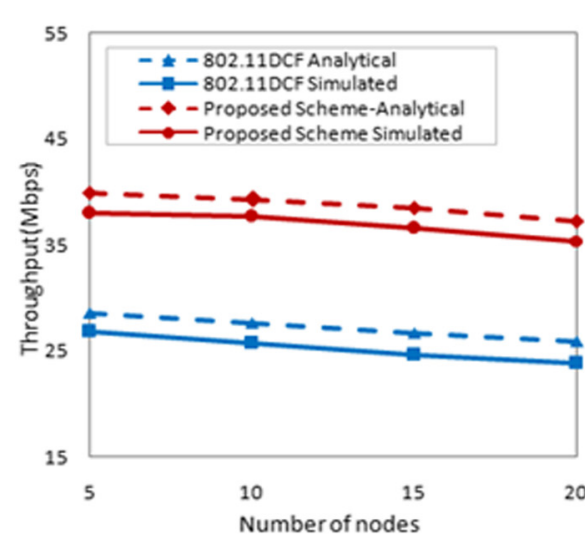

(a)

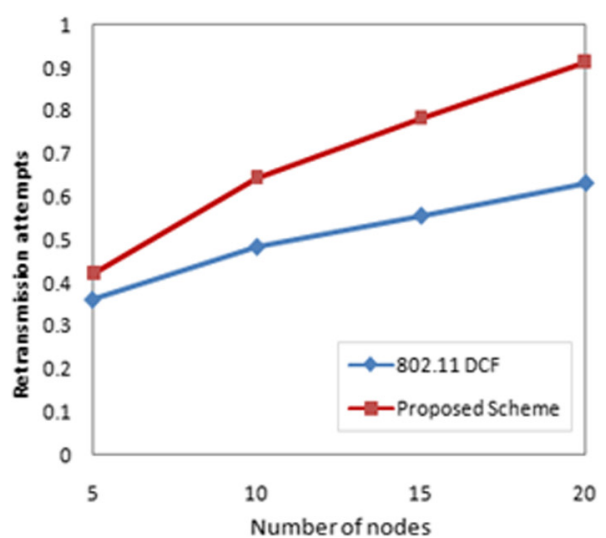

(b)

Figure 8 Simulation results. (a) Network throughput and (b) retransmission attempts. 
on the same channel. The network topology is given in Figure 7, where the mobility of stations are not assumed. The numbers of member stations in both BSSs are the same and all the stations periodically transmit to AP the constant bit rate (CBR) UDP packets which are 1,024 bytes long. The IEEE 802.11a is adopted for the WLAN proto$\mathrm{col}$, and other parameters are presented in Table 2 . All the results reported here are the average values of 20 runs.

Figure 8a shows that the overall network throughput is inversely proportional to the increment of the network size, where the number of member stations in each BSS varies from 5 to 20 . We can see that the simulation result closely follows the analytic data and our proposed scheme enhances the overall network performance. Note that as the stations in legacy 802.11 MAC contend for accessing channel with the stations in another BSS, one BSS is allowed to transmit. The proposed DTPC, however, enables the stations to dynamically adjust their transmit powers such that other stations in different BSSs can have more opportunities of simultaneous transmissions.

Figure $8 \mathrm{~b}$ presents the retransmission attempts versus the network size. We find the retransmission attempts in our proposed scheme are lower than that of the legacy one. It can be achieved by the fact that our proposed scheme enables RTS/CTS frames to be transmitted on a maximum power and it prevents hidden and exposed terminals occurring in a wide range. In addition, unlike the legacy scheme, the neighbor AP can adjust its power to a proper level such that it can respond with immediate ACKs.

\section{Conclusions}

In this paper, we have presented a dynamic transmit power control scheme, namely DTPC, for enhancing the performance of OBSSs. Stations can dynamically adjust their transmit powers using the proposed DTPC, which enables the overlapping BSSs to transmit simultaneously and to enhance the spatial reuse. We have developed a Markov chain model in order to analyze the performance of DTPC, and the simulation results prove that the analytical model is properly built. Both analytic and simulation results show that the proposed DTPC significantly improves the performance of OBSSs. As the future work, we plan to investigate the performance of DTPC operating in multiple OBSSs rather than two OBSSs. Also the hidden and/or exposed terminals existed in a BSS and various network topologies should be studied as a future work.

Competing interests

The authors declare that they have no competing interests.

\section{Acknowledgements}

This work was supported by Basic Science Research Program through the National Research Foundation of Korea (NRF) funded by the Ministry of Education, Science and Technology (2013008855), and in part by the Research Grant of Kwangwoon University in 2013
Received: 26 February 2014 Accepted: 22 December 2014

Published online: 24 January 2015

\section{References}

1. W Li, Y Cui, X Cheng, MA Al-Rodhaan, A Al-Dhelaan, Achieving proportional fairness via AP power control in multi-rate WLANs. IEEE Trans. Wireless Commun. 10(11), 3784-3792 (2011)

2. A Jow, C Schurgers, Borrowed channel relaying: a novel method to improve infrastructure network throughput. EURASIP J. Wireless Commun. Netw. 2009174730 (2010)

3. B Han, L Ji, S Lee, RR Miller, B Bhattacharjee, in IEEE International Conference on Communications. Channel access throttling for overlapping BSS management (Dresden, 2009), pp. 1-6

4. O Oteri, P Xia, F LaSita, R Olesen, in 16th International Symposium on Wireless Personal Multimedia Communications, WPMC. Advanced power control techniques for interference mitigation in dense 802.11 networks (Atlantic, 2013), pp. 1-7

5. X Wang, H Lou, M Ghosh, G Zhang, P Xia, O Oteri, F La Sita, R Olesen, N Shah, in Systems, Applications and Technology Conference, LISAT, 2014 IEEE Long Island. Carrier grade Wi-Fi: air interface requirements and technologies (Farmingdale, 2014), pp. 1-6

6. X Liu, S Seshan, P Steenkiste, in proceedings of the annual conference of ITA Interference-aware transmission power control for dense wireless networks, (2007), pp. 1-7

7. H Ma, J Zhu, S Roy, SY Shin, Joint transmit power and physical carrier sensing adaptation based on loss differentiation for high density IEEE 802.11 WLAN. Comput. Netw. 52, 1703-1720 (2008)

8. M Cesana, D Maniezzo, P Bergamo, M Gerla, in Vehicular Technology Conference 2003. Interference aware (IA) MAC: an enhancement to IEEE802.11b DCF (Orlando, Florida, USA, 2003), pp. 2799-2803

9. D Qiao, S Choi, A Jain, KG Shin, in Proceedings of the 9th annual international conference on mobile computing and networking. MiSer: an optimal low energy transmission strategy for IEEE 802.11a/h (San Diego, California, USA, 2003), pp. 161-175

10. X Wang, GB Giannakis, CSMA/CCA: a modied CSMA/CA protocol mitigating the fairness problem for IEEE 802.11 DCF. EURASIP J. Wireless Commun. Netw. 2006, 039604 (2006)

11. Y Li, X Wang, SA Mujtaba, in Vehicular Technology Conference, 2003. Co-channel interference avoidance algorithm in 802.11 wireless LANs (Orlando, Florida, USA, 2003), pp. 2610-2614

12. Y Fang, D Gu, AB McDonald, J Zhang, in The 14th IEEE Workshop on Local and Metropolitan Area Networks. Two-level carrier sensing in overlapping basic service sets (BSSs) (Island of Crete, Greece, 2005), p. 6

13. T Tandai, K Toshimitsu, T Sakamoto, in Personal, Indoor and Mobile Radio Communications, 2006. Interferential packet detection scheme for a solution to overlapping BSS issues in IEEE 802.11 WLANs (Finland, 2006), pp. $1-5$

14. IEEE 802.11-2012. Part 11, Wireless LAN medium access control (MAC) and physical layer (PHY) specifications. IEEE (2012)

15. J Mo, H-S So, J Walrand, Comparison of multichannel MAC protocols. IEEE Trans. Mobile Comput. 7(1), 60-65 (2008)

\section{Submit your manuscript to a SpringerOpen ${ }^{\circ}$ journal and benefit from:}

- Convenient online submission

- Rigorous peer review

- Immediate publication on acceptance

- Open access: articles freely available online

- High visibility within the field

- Retaining the copyright to your article

Submit your next manuscript at $>$ springeropen.com 